\title{
Paramètres influençant la distribution des éléments traces métalliques dans les affluents du fleuve Garonne (France)
}

\author{
Yao Mathieu N'GUESSAN ${ }^{1 *}$, Valérie Ama WOGNIN ${ }^{1}$, Kouamé Georges KOUADIO ${ }^{2}$, \\ Anne PROBST ${ }^{3}$ et Jean Luc PROBST ${ }^{3}$
}

${ }^{1}$ Laboratoire de Géochimie et Environnement, Département des Géosciences Marines, UFR STRM, Université Félix Houphouët-Boigny, Cocody, 22 B.P. 582 Abidjan 22, Côte d'Ivoire.

${ }^{2}$ Département des Sciences et Technologies, Ecole Normale Supérieure d'Abidjan, 08 B.P. 10 Abidjan 08, Côte d'Ivoire.

${ }^{3}$ Laboratoire d'Ecologie Fonctionnelle et Environnement (EcoLab), ENSAT, INPT, UPS,

Université de Toulouse, Centre National de la Recherche Scientifique (CNRS), Toulouse, France. *Auteur correspondant ; E-mail :nguessym@yahoo.fr

\section{RESUME}

Les affluents de la rive gauche du fleuve Garonne, dans le Sud-Ouest de la France, traversent des bassins versants marqués par de fortes activités agricoles. Pour évaluer la qualité de la phase dissoute de ces cours d'eau, soixante-quinze (75) échantillons d'eau ont été prélevés. Plusieurs éléments traces métalliques ont été analysés dans cette phase aqueuse. Les concentrations moyennes de ces éléments, dans cette région, sont dans l'ordre décroissant : Zn $\left(4,46 \mu \mathrm{g} .1^{-1}\right)$, As $\left(1,03 \mu \mathrm{g} .1^{-1}\right), \mathrm{Cu}\left(0,79 \mu \mathrm{g} . \mathrm{l}^{-1}\right), \mathrm{Ni}\left(0,62 \mu \mathrm{g} . \mathrm{l}^{-1}\right), \mathrm{Cr}\left(0,30 \mu \mathrm{g} . \mathrm{l}^{-1}\right)$, $\mathrm{Co}\left(0,23 \mu \mathrm{g} \cdot 1^{-1}\right), \mathrm{Pb}\left(0,04 \mu \mathrm{g} \cdot 1^{-1}\right)$ et $\mathrm{Cd}\left(0,01 \mu \mathrm{g} \cdot 1^{-1}\right)$. Ces valeurs sont, pour la plupart, inférieures à celles des eaux de rivières des zones non anthropisées à l'échelle mondiale. La distribution de ces polluants, dans les écosystèmes aquatiques de cette région, est notamment contrôlée par les chlorures et le carbone organique dissous, surtout dans les zones où la contribution anthropique en chlorures et en matière organique est très significative. Dans les zones moins anthropisées, la disponibilité de ces éléments traces métalliques, dans la colonne d'eau, diminue lorsque le $\mathrm{pH}$ et l'alcalinité augmentent conformément aux processus géochimiques naturels. Ces observations traduisent l'action prépondérante des activités anthropiques sur la mobilité et la disponibilité biologique des polluants métalliques dans les affluents du fleuve Garonne.

(C) 2017 International Formulae Group. All rights reserved.

Mots Clés : Fleuve Garonne, éléments traces métalliques, phase dissoute, distribution.

\section{Parameters influencing metal trace elements distribution in the tributaries of the Garonne River (France)}

\begin{abstract}
The left-bank tributaries of the Garonne River, located in the South-West of France, cross catchments, is affected by significant agricultural activities. To evaluate the quality of the dissolved fraction, seventy-five (75) samples of river waters were collected. Several trace elements were analyzed in this aqueous phase. The average metal trace element concentrations, in this region, are in the decreasing order: $\mathrm{Zn}\left(4.46 \mu \mathrm{g} . \mathrm{l}^{-1}\right)$, As
\end{abstract}


$\left(1.03 \mu \mathrm{g} .1^{-1}\right), \mathrm{Cu}\left(0.79 \mu \mathrm{g} .1^{-1}\right) \mathrm{Ni}\left(0.62 \mu \mathrm{g} .1^{-1}\right), \mathrm{Cr}\left(0.30 \mu \mathrm{g} .1^{-1}\right)$, Co $\left(0.23 \mu \mathrm{g} .1^{-1}\right), \mathrm{Pb}\left(0.04 \mu \mathrm{g} .1^{-1}\right)$, and Cd $(0.01$ $\left.\mu \mathrm{g} .1^{-1}\right)$. These values are, for the majority, lower than those of waters of rivers in non-anthropized areas. The distribution of these elements is controlled by chloride and organic matter, particularly in the areas where the anthropogenic inputs of these compounds are significant. In less anthropized areas, the availability of these trace elements in the water column decreases when the $\mathrm{pH}$ and the alkalinity increase according to natural geochemical processes. These observations show the predominant action of anthropogenic activities on the mobility and the biological availability of metallic pollutants in the tributaries of the Garonne River.

(C) 2017 International Formulae Group. All rights reserved.

Keywords: Garonne River; metal trace elements; dissolved phase; distribution.

\section{INTRODUCTION}

Les éléments traces ne représentent que $0,6 \%$ du total des éléments chimiques de la croûte terrestre. Cependant, leurs effets sur les écosystèmes terrestres sont souvent dévastateurs (Kabata-Pendias et Mukherjee, 2007 ; Soro et al., 2009). Contrairement aux polluants organiques, les éléments traces métalliques ne peuvent pas être dégradés biologiquement et chimiquement (Senou et al., 2012). Une des conséquences les plus sérieuses de la persistance des métaux est leur accumulation dans les chaînes alimentaires (Mélila et al., 2012 ; Aduayi-Akue et Gnandi, 2014). Au bout de ces chaînes, les éléments traces peuvent atteindre des concentrations 10 fois supérieures à celles trouvées dans l'eau (Bliefert et Perraud, 2001).

Ces éléments et leurs composées sont souvent transportés sur de grandes distances par l'eau avec ou sans modification chimique. Les réactions de transformation conduisent généralement à des composés plus toxiques que l'élément d'origine (par exemple, la méthylation $\mathrm{du}$ mercure). En revanche, l'immobilisation des éléments traces (par exemple, la précipitation de $\mathrm{PbSO}_{4}$ ) par les particules diminue leur disponibilité pour les organismes aquatiques (Devallois, 2009; N'guessan et al., 2016).

A l'échelle des bassins versants agricoles, les processus et phénomènes majeurs qui vont influencer l'apport et le devenir de ces éléments traces sont l'altération, l'érosion, le ruissellement, les paramètres physico-chimiques des milieux et enfin les actions des pratiques agricoles. En France, six zones géographiques correspondant à des bassins versants naturels ont été délimitées dans le cadre de la politique de gestion et de suivi des milieux aquatiques. L'une de ces six zones est le bassin Adour-Garonne qui occupe tout le quart Sud-Ouest de la France.

$\mathrm{Au}$ niveau hydrologique, le bassin Adour-Garonne présente un réseau hydrographique dense et varié. Ce bassin est traversé par la Garonne, qui est le troisième fleuve français en terme de débit. Pour ce qui est des caractéristiques anthropiques, cette région abrite deux grandes métropoles, Toulouse et Bordeaux, génératrices d'effluents domestiques et industriels, ainsi que des rejets atmosphériques par l'intermédiaire des industries, des incinérateurs et du parc automobile. A cela, il faut ajouter la forte activité agricole dans cette région de la France, avec son corollaire d'impacts liés aux fertilisants, aux pesticides, aux boues de stations d'épuration et aux actions des engins agricoles. Enfin, il faut noter que cette zone abrite d'anciens sites miniers désaffectés dans le Massif pyrénéen et dans le Massif central.

La Gascogne est soumise à un climat océanique, caractérisée par un hiver doux et humide, un été sec et chaud, et une pluviométrie comprise entre 700 et $1000 \mathrm{~mm}$ par an. La température moyenne annuelle est comprise entre 12 et $13{ }^{\circ} \mathrm{C}$, avec janvier comme mois le plus froid, (moyenne varie entre 5 et $6{ }^{\circ} \mathrm{C}$ ) et août comme mois le plus chaud, (moyenne comprise entre 20 et $21^{\circ} \mathrm{C}$ ). Toutes les caractéristiques géologiques, climatiques, morphologiques, pédologiques, géographiques, hydrologiques et anthropiques font de cette région une zone particulièrement vulnérable aux pollutions. 
C'est la raison pour laquelle depuis une vingtaine d'année, au travers de différents programmes de recherches, plusieurs travaux (Baque, 2006; Perrin et al., 2008 ; Bur et al., 2009 ; N'guessan et al., 2009 ; Oeurng et al., 2010; Taghavi, 2010) portant sur les aspects biologique, géochimique, sociologique et économique, ont été menés sur le bassin Adour-Garonne. Les derniers en date sont ceux du groupement ECOBAG "Environnement, Ecologie et Economique du Bassin Adour Garonne". Ces programmes regroupent l'ensemble des acteurs environnementaux du bassin, à savoir gestionnaires publics, privés, scientifiques, en vue d'une meilleure gestion $d u$ bassin à travers plusieurs approches transversales.

Les travaux de la présente étude s'insèrent dans le cadre de ce groupement et ont été développés dans le cadre du projet IMAQUE (Impacts des Activités Anthropiques sur la qualité des eaux, des sols, des sédiments et des milieux aquatiques). Ces travaux s'intéressent particulièrement aux cours d'eau des bassins versants de la Gascogne, qui présentent la particularité d'être les principaux fournisseurs d'eau et de matières de la rive gauche du fleuve Garonne. Cette étude vise à estimer avec précision le contenu de la phase dissoute en éléments traces métalliques et à déterminer les paramètres de contrôle de ces polluants dans un contexte de fortes activités agricoles et de milieu carbonaté.

\section{MATERIELS ET METHODES}

Prélèvement et conditionnement des eaux de surface

Sur la rive gauche du fleuve Garonne, vingt-cinq (25) stations de prélèvement ont été positionnées, pour la réalisation de cette étude (Figure 1). Les échantillons d'eau ont été prélevés, au cours de trois différentes campagnes saisonnières (Hiver, Printemps et Eté), à l'aide d'un sceau, à partir d'un pont ou d'un endroit qui nous évite de descendre dans la rivière et de troubler l'eau, car la représentativité de colonne d'eau pourrait être influencée. L'eau recueillie est ensuite versée dans des bidons de 2 litres préalablement rincés à l'eau de la rivière. Ceux-ci sont soigneusement fermés et conservés au frais sur le terrain dans une glaciaire, puis au laboratoire en chambre froide jusqu'à la phase de filtration.

En plus des échantillons d'eau, certains paramètres physico-chimiques $(\mathrm{pH}$, température et conductivité) des cours d'eau ont été mesurés directement sur le terrain. Afin d'éviter une modification significative de ces paramètres due aux échanges éventuelles que pourraient avoir les échantillons avec l'extérieur, ces mesures sont faites sitôt : l'échantillon prélevé à l'aide d'un thermomètre à sonde de platine pour la température, d'un $\mathrm{pH}$ mètre portable KNICK 651 étanche équipé d'une électrode combinée pour la détermination du $\mathrm{pH}$, et d'un conductimètre associé à une électrode pour la conductivité de l'eau. Il est nécessaire d'étalonner le pH-mètre: deux étalons ont servi à calibrer l'appareil aux environ de $\mathrm{pH}$ 7,0 et 5,0 ou à 9,0 selon le secteur de prélèvement. Ces étalons, maintenus à la température de la source, permettent de mesurer le $\mathrm{pH}$ avec exactitude pour toute température. La lecture se fait immédiatement en terme de $\mathrm{pH}$, à 0,01 unité près. Pour la conductivité, la calibration s'effectue avec une solution étalon de $1278 \mu \mathrm{S} . \mathrm{cm}^{-1}$.

\section{Traitement physique et chimique des échantillons}

Les dosages d'éléments traces dans l'eau nécessitent, au préalable, la filtration de la solution pour supprimer toute suspension susceptible de contenir des contaminants organiques hydrophobes et des métaux traces dont les teneurs sont souvent plus de 1000 fois supérieures à la concentration dans la phase dissoute (Quevauviller, 2001). Les échantillons d'eau de cette étude ont donc été filtrés grâce à des filtres MILLIPORE de porosité $0,45 \mu \mathrm{m}$ qui est une barrière internationale permettant de distinguer la phase dissoute $(<0,45 \mu \mathrm{m})$ de la phase particulaire $(>0,45 \mu \mathrm{m})$. Les filtres sont préalablement trempés pendant 3 jours dans du $\mathrm{HCl}(25 \%)$ et abondamment rincés avec de l'eau déminéralisée avant leur emploi. Les 
filtrats sont ensuite récupérés dans différents piluliers, selon le type d'analyse envisagé. Un traitement à l'acide nitrique bidistillé est réalisé sur les filtrats destinés à la mesure des cations majeurs et des éléments traces. Cette acidification est fortement recommandée et permet ainsi d'éviter les précipitations, les phénomènes d'adsorption/désorption à partir des colloïdes, ou d'autres réactions d'ordre biochimique. De même le filtrat destiné à la mesure du Carbone Organique Dissout (COD) subit un traitement à l'acide chlorhydrique pour bloquer l'activité bactérienne. Les échantillons sont conservés entre 3 et $5{ }^{\circ} \mathrm{C}$ à l'abri de la lumière.

\section{Méthodes d'analyse des éléments traces}

Les solutions obtenues par filtration ont été analysées à l'ICP-MS au Laboratoire des Mécanismes et des Transferts en Géologie (LMTG) de Toulouse. Avant tout dosage d'éléments traces à l'Inductively Coupled Plasma Mass Spectrometry (ICP-MS), quelques gouttes de standard interne (Indium/Rhénium) sont ajoutées aux échantillons pour s'affranchir des effets de matrice (baisse de la qualité du signal) et des dérives temporelles liées à la machine.
L'efficacité du protocole de filtration a été évaluée respectivement à l'aide d'une solution de rivière certifiée (SLRS-4). Le matériau de référence a été traité identiquement et dans les mêmes conditions que les échantillons. En plus de ce matériau, des blancs de filtration ont également été analysés pour vérifier toute contamination à l'environnement et aux matériels de travail. Enfin, pour la précision analytique, un coefficient de variation a été déterminé pour chaque élément; coefficient qui se définit comme l'écart-type rapporté à la moyenne (Relative Standard Deviation: RSD, en \%).

Les résultats de la SLRS 4 analysé, rapportés aux valeurs certifiées (Tableau 1) montrent un recouvrement compris entre $78 \%$ et $138 \%$. Les limites de détection et de quantification de l'ICP-MS ont été assez faibles au cours des analyses, ce qui est un bon signe pour la qualité des valeurs mesurées sur les échantillons. Aussi, les valeurs de la RSD sont inférieures à $10 \%$, à l'exception de $\mathrm{Cd}(15 \%)$ qui présente, à l'opposé des autres éléments, des concentrations proches de la limite de détection. Selon Roussiez (2005) et Pekey (2006) cette proximité de ces valeurs pourrait expliquer ce pourcentage élevé de RSD pour Cd.

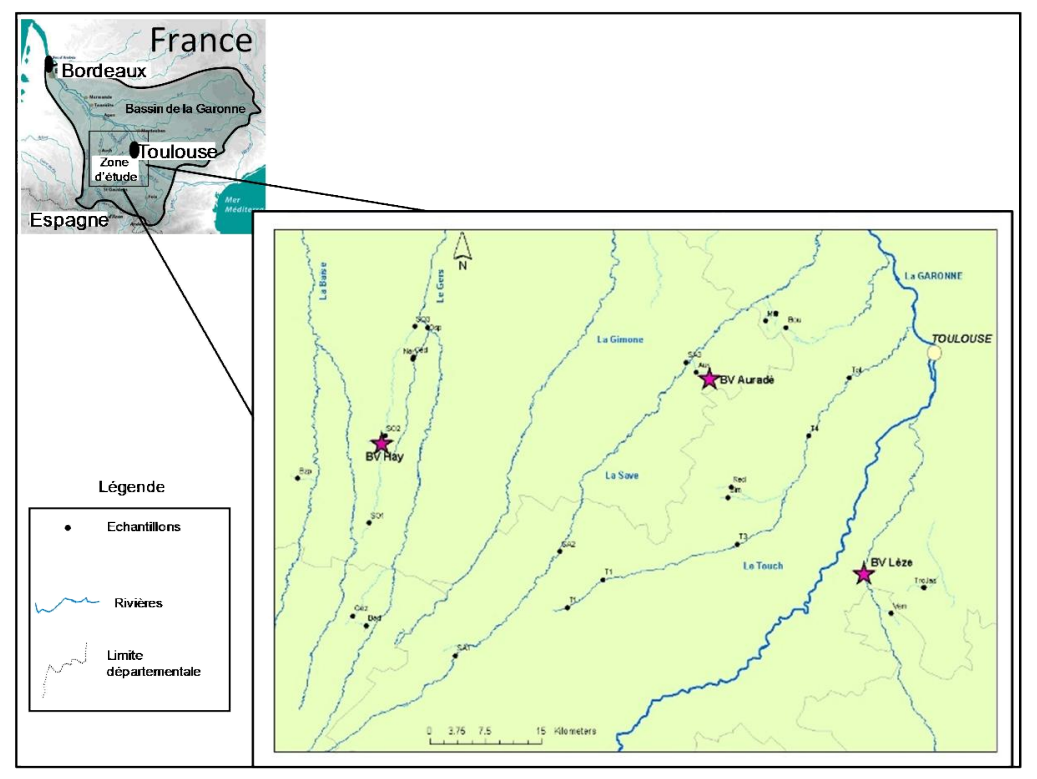

Figure 1 : Localisation des différents points d'échantillonnage sur la rive gauche de la Garonne. 
Tableau 1 : Précision analytique et reproductibilité du protocole de filtration des échantillons d'eau.

\begin{tabular}{|c|c|c|c|c|c|c|}
\hline Eléments & $\begin{array}{l}\text { SLRS } 4^{\mathrm{a}} \\
\text { mesuré } \\
\left(\mu \mathrm{g} . \mathrm{L}^{-1}\right)\end{array}$ & $\begin{array}{c}\text { SLRS } 4^{\mathrm{a}} \\
\text { certifié } \\
\left(\mu \mathrm{g.L} \mathrm{L}^{-1}\right)\end{array}$ & $\begin{array}{c}\text { Recouvrement } \\
(\%)\end{array}$ & $\begin{array}{c}\text { RSD }^{b} \\
(\%)\end{array}$ & $\begin{array}{c}\text { Limite de } \\
\text { détection } \\
\left(\mu \mathrm{g} . \mathrm{L}^{-1}\right)\end{array}$ & $\begin{array}{c}\text { Limite de } \\
\text { quantification } \\
\left(\mu \mathrm{g} . \mathrm{L}^{-1}\right)\end{array}$ \\
\hline $\mathrm{Sc}$ & 0,662 & $*$ & - & 5,2 & 0,47 & 1,568 \\
\hline $\mathrm{Cr}$ & 0,358 & 0,330 & 108 & 10,2 & 0,13 & 0,432 \\
\hline Co & 0,040 & 0,033 & 122 & 6,9 & 0,005 & 0,015 \\
\hline $\mathrm{Ni}$ & 0,713 & 0,670 & 106 & 9,1 & 0,03 & 0,095 \\
\hline $\mathrm{Cu}$ & 1,883 & 1,810 & 104 & 5,1 & 0,01 & 0,033 \\
\hline $\mathrm{Zn}$ & 1,279 & 0,930 & 138 & 4,9 & 0,03 & 0,100 \\
\hline As & 0,771 & 0,680 & 113 & 4,7 & 0,07 & 0,219 \\
\hline Cs & 0,007 & 0,009 & 78 & 6,9 & 0,003 & 0,009 \\
\hline $\mathrm{Pb}$ & 0,075 & 0,086 & 88 & 6,7 & 0,001 & 0,003 \\
\hline $\mathrm{Cd}$ & 0,016 & 0,012 & 131 & 15,2 & 0,008 & 0,032 \\
\hline
\end{tabular}

${ }^{a}$ Materiau de référence, SLRS 4 (Eau du fleuve Saint-Laurent, National Research Council Canada), ${ }^{b}$ Relative Standard Deviation, ${ }^{*}$ non certifié.

\section{RESULTATS}

Abondance et variabilité des éléments traces métalliques dans la phase dissoute

Dans les eaux de la région (rive gauche

de la Garonne), l'élément trace le plus abondant est le $\mathrm{Zn}$, marqué par une concentration moyenne de 4,46 $\mu \mathrm{g} . \mathrm{l}^{-1}$. De manière décroissante, l'abondance des autres éléments s'établit comme suit: As $\left(1,03 \mu \mathrm{g} . \mathrm{l}^{-}\right.$ $\left.{ }^{1}\right), \mathrm{Cu}\left(0,79 \mu \mathrm{g} .1^{-1}\right), \mathrm{Ni}\left(0,62 \mu \mathrm{g} .1^{-1}\right), \mathrm{Cr}(0,30$ $\left.\mu \mathrm{g} .1^{-1}\right)$, Co $\left(0,23 \mu \mathrm{g} .1^{-1}\right)$, et enfin $\mathrm{Pb}$ et $\mathrm{Cd}$ qui, avec des teneurs respectives de $0,04 \mu \mathrm{g} .1^{-1}$ et $0,01 \mu \mathrm{g} . \mathrm{l}^{-1}$, sont en moyenne plus de 10 fois moins concentrés que les autres éléments traces (Tableau 2). Ces concentrations en éléments traces intègrent les valeurs établies pour les eaux de rivières naturelles du monde (Meybeck et Helmer, 1989). Seules les valeurs de $\mathrm{Cd}$ sont supérieures à celles de ces rivières dites non polluées.

Dans l'ensemble (Figure 2), les plus fortes concentrations en éléments traces des phases dissoutes de la région se rencontrent au niveau de la station «Bou », à l'exception de $\mathrm{Zn}$ et de $\mathrm{Cr}$ qui présentent, respectivement, leur maxima à « TO2 » et à «Vem ».

Les courbes d'évolution du plomb et du nickel montrent quelques similarités, caractérisées par des pics au niveau de «Bou » ( $\mathrm{au}$ Nord de Toulouse) et de " Gez » ( $\mathrm{au}$ Sud-Ouest de Toulouse), et de très faibles concentrations au niveau de « Mar » (au Nord de Toulouse).

Les plus fortes concentrations en cobalt sont à « Bou », et à un degré moindre à « Red » (au Sud de Toulouse), « Hay » et « SO2 » (à l'Ouest de Toulouse), et à TO2 (sur le Touch, au centre de la zone d'étude). Les plus faibles valeurs sont observées à SA1 (amont de la Save) et à Bad (au Sud-Ouest).

En dehors de la station «Bou » (marquée par de fortes concentrations en $\mathrm{Cu}$ et $\mathrm{Zn}$ ), le cuivre et le zinc dissous montrent des différences notables sur l'ensemble de la zone 
d'étude. Alors que $\mathrm{Cu}$ présente de fortes concentrations sur le Sousson (notamment au niveau de « SO2 »), les valeurs en zinc sur ce ruisseau font partie des plus faibles de la région. A l'inverse, sur le Touch (précisément au niveau de "TO2 ») où le zinc a sa plus forte valeur, le cuivre présente, par contre, sa plus faible teneur.

L'arsenic, en plus du pic à « Bou», montre quelques pics sur le Sousson (SO2), le Touch (TO2 et TO4), ainsi que sur le Cedon et le Vermeil. Sa plus faible teneur se situe au Sud-Ouest, précisément au niveau de « Gez ».

La courbe d'évolution du chrome indique quelques importantes variations bien surprenantes. Par exemple, les stations «Vem » (au Sud de Toulouse) et Mar (au Nord de Toulouse) montrent respectivement des teneurs en chrome 17 et 12 fois supérieures à celles mesurées à " Aur », alors que ces trois stations sont fortement agricoles. Entre « Gez » et « Sim », stations sous forêt et situées au Sud de Toulouse, on note également des différences relativement significatives, avec un facteur de variation de 3 .
Relations entre les éléments traces de la phase dissoute

Le Tableau 3 montre des relations plus ou moins significatives entre les éléments traces dissous (excepté $\mathrm{Cr}$ ). Parmi ces éléments, $\mathrm{Co}, \mathrm{Ni}, \mathrm{Pb}$ et $\mathrm{Cd}$ présentent d'assez importantes corrélations $\left(\mathrm{r}^{2}>0,84\right)$. La représentation graphique de certaines de ces relations (Figure 3) souligne le poids des stations, Bou et Gez, dans les valeurs de $r^{2}$ obtenues. En effet, lorsqu'on isole ces deux stations, les relations deviennent moins significatives entre $\mathrm{Cd}, \mathrm{Pb}$ et $\mathrm{Ni}$, mais également entre tous les éléments (Tableau 3). Seuls Ni et Co conservent toujours une bonne relation $\left(r^{2}=0,7\right)$.

Ces résultats traduisent la complexité à établir des relations significatives entre les éléments traces de la phase dissoute en dehors des stations « Bou » et " Gez ». Le caractère commun de ces deux stations est leur couverture forestière qui est relativement importante. Par contre, elles se distinguent par leurs teneurs en éléments majeurs (notamment $\mathrm{Cl}^{-}$et $\mathrm{Na}^{+}$) et en carbone organique dissous, qui sont assez importantes pour «Bou " (N'guessan et al., 2016).

Tableau 2: Teneurs en éléments traces dissous dans les cours d'eau alimentant la Garonne, comparées à l'ITD et aux valeurs des rivières du monde.

\begin{tabular}{|c|c|c|c|}
\hline \multicolumn{2}{|c|}{ Les affluents de la Garonne } & \multirow{2}{*}{$\begin{array}{c}\begin{array}{c}\text { Concentration naturelle } \\
\text { des rivières du monde }\end{array} \\
\text { Moy. }\left(\mu \mathrm{g} . \mathrm{I}^{-1}\right) \\
\end{array}$} & \multirow{2}{*}{$\begin{array}{c}\text { Index de transport dissous }{ }^{\mathrm{b}} \\
\% \\
\end{array}$} \\
\hline Eléments traces & Moy. $\left(\mu \mathrm{g} . \mathrm{I}^{-1}\right)$ & & \\
\hline $\mathrm{Cr}$ & 0,30 & $0,1-1$ & 2,5 \\
\hline Co & 0,23 & 0,2 & 2,5 \\
\hline $\mathbf{N i}$ & 0,62 & 2,2 & 5 \\
\hline $\mathrm{Cu}$ & 0,79 & $1,4-12,4$ & 19 \\
\hline $\mathbf{Z n}$ & 4,46 & $0,2-37,5$ & 17 \\
\hline As & 1,03 & $0,5-2$ & 44 \\
\hline Cd & 0,01 & $0,001-0,005$ & - \\
\hline $\mathbf{P b}$ & 0,04 & $0,04-4$ & 2 \\
\hline
\end{tabular}

Moy $=$ moyenne. ${ }^{a}$ Meybeck et Helmer (1989); ${ }^{b}$ Martin et Meybeck (1979). 
$\mathrm{Cr}$

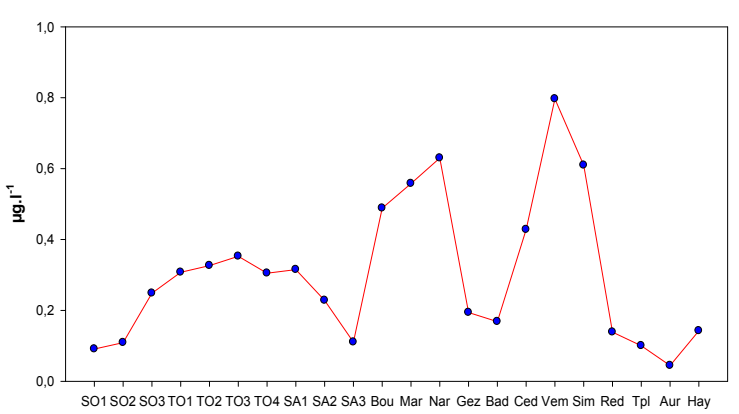

$\mathrm{Ni}$

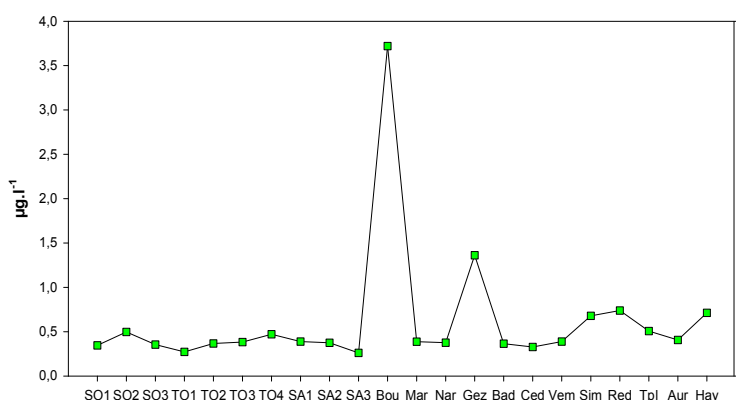

$\mathrm{Zn}$

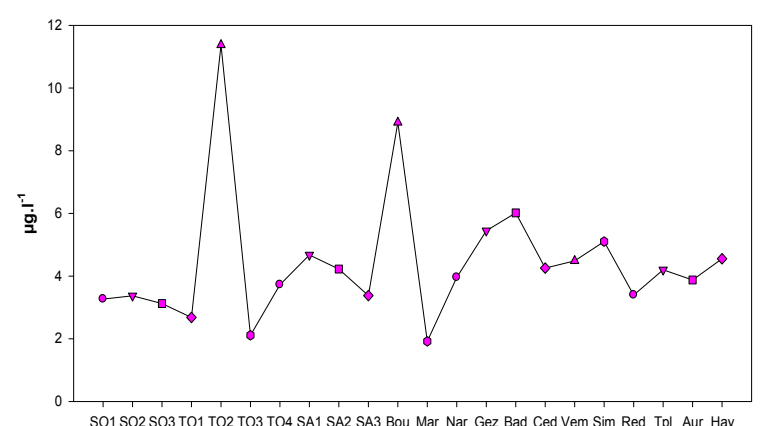

Cd

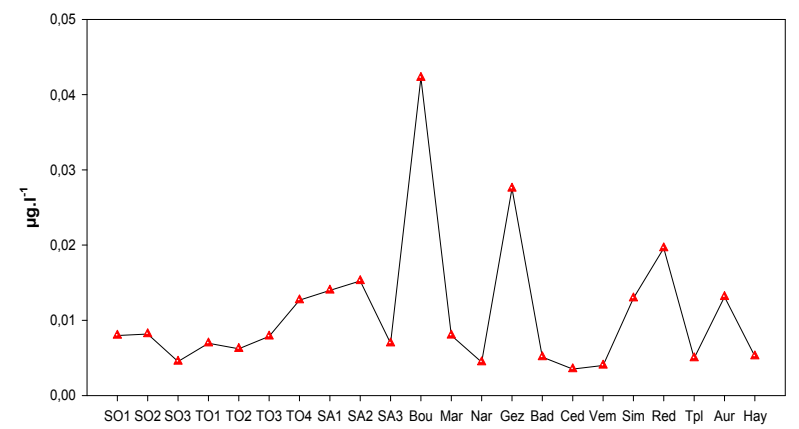

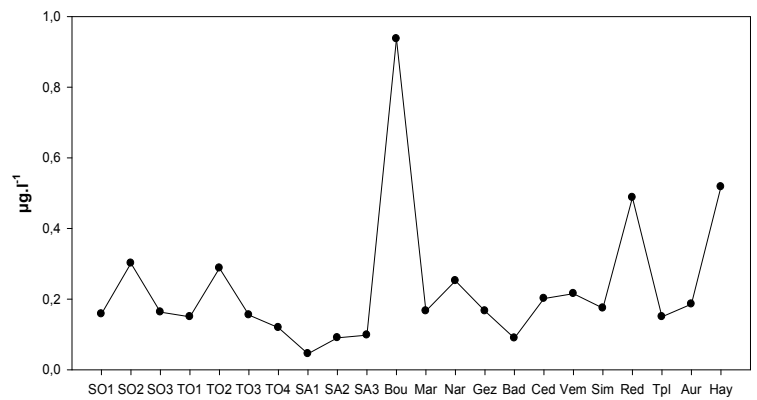

$\mathrm{Cu}$

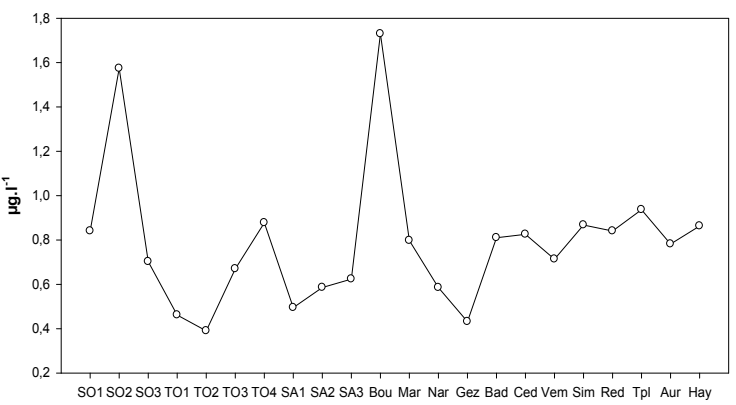

As

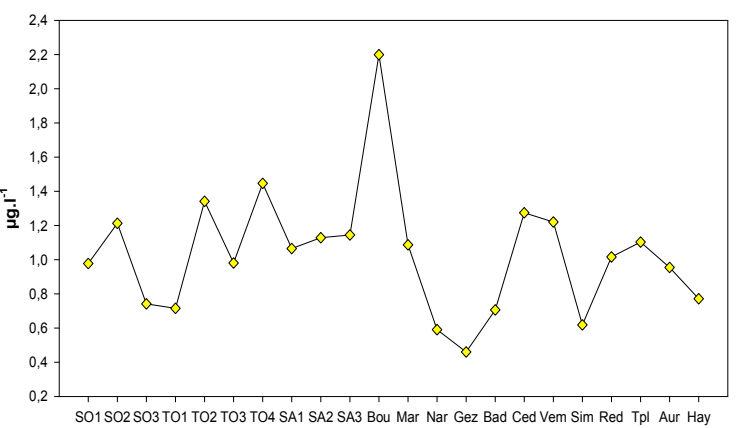

$\mathrm{Pb}$

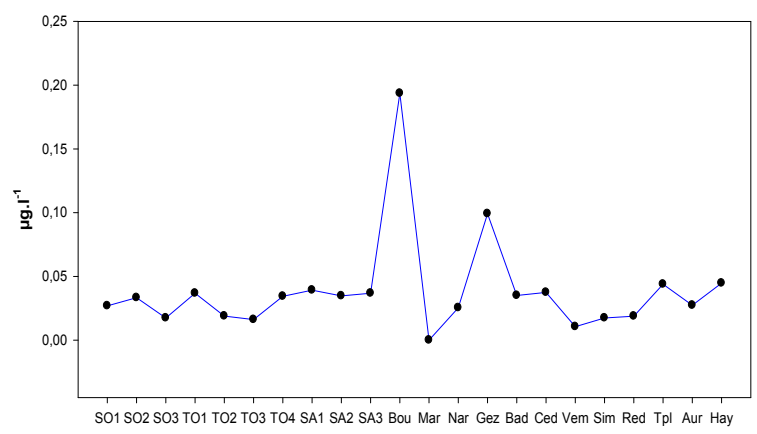

Figure 2 : Variabilité spatiale des concentrations en éléments traces dissous dans les affluents de la rive gauche de la Garonne. 
Tableau 3: Coefficient de corrélation $\left(\mathrm{r}^{2} ; \mathrm{p}<0,001\right)$ entre les éléments traces de la phase dissoute $(\mathrm{n}=22)$.

\begin{tabular}{|c|c|c|c|c|c|c|c|c|}
\hline & $\mathrm{Cr}$ & Co & $\mathbf{N i}$ & $\mathbf{C u}$ & $\mathbf{Z n}$ & As & Cd & $\mathbf{P b}$ \\
\hline $\mathrm{Cr}$ & 1 & $0,11 /-0,09 *$ & $0,15 /-0,08^{*}$ & $-0,03 /-0,26^{*}$ & $0,11 / 0,04^{*}$ & $0,13 /-0,08^{*}$ & $0,01 /-0,23^{*}$ & $-0,02 /-\mathbf{0}, \mathbf{5} 7^{*}$ \\
\hline Co & & 1 & $0,82 / 0,70^{*}$ & $\mathbf{0 , 6 5} / 0,28^{*}$ & $0,44 / 0,13^{*}$ & $\mathbf{0 , 5 5} /-0,09 *$ & $\mathbf{0 , 6 1} / 0,05^{*}$ & $\mathbf{0 , 6 8} /-0,06^{*}$ \\
\hline $\mathbf{N i}$ & & & 1 & $\mathbf{0 , 6 1 / 0 , 4 3 *}$ & $0,48 / 0,03 *$ & $\mathbf{0 , 5 7 / - 0 , 1 6 ^ { * }}$ & $\mathbf{0 , 8 8} / 0,43^{*}$ & $\mathbf{0 , 9 3 / 0 , 0 1 *}$ \\
\hline $\mathrm{Cu}$ & & & & 1 & $0,09 /-0,29^{*}$ & $\mathbf{0 , 5 9} / 0,14^{*}$ & $0,41 / 0,01 *$ & $\mathbf{0 , 5 1} / 0,11^{*}$ \\
\hline $\mathbf{Z n}$ & & & & & 1 & $0,40 / 0,19^{*}$ & $0,37 /-0,11^{*}$ & $0,47 / 0,06^{*}$ \\
\hline As & & & & & & 1 & $0,44 / 0,12 *$ & $\mathbf{0 , 5 0} / 0,02 *$ \\
\hline Cd & & & & & & & 1 & $\mathbf{0 , 8 4} /-0,05^{*}$ \\
\hline $\mathbf{P b}$ & & & & & & & & 1 \\
\hline
\end{tabular}
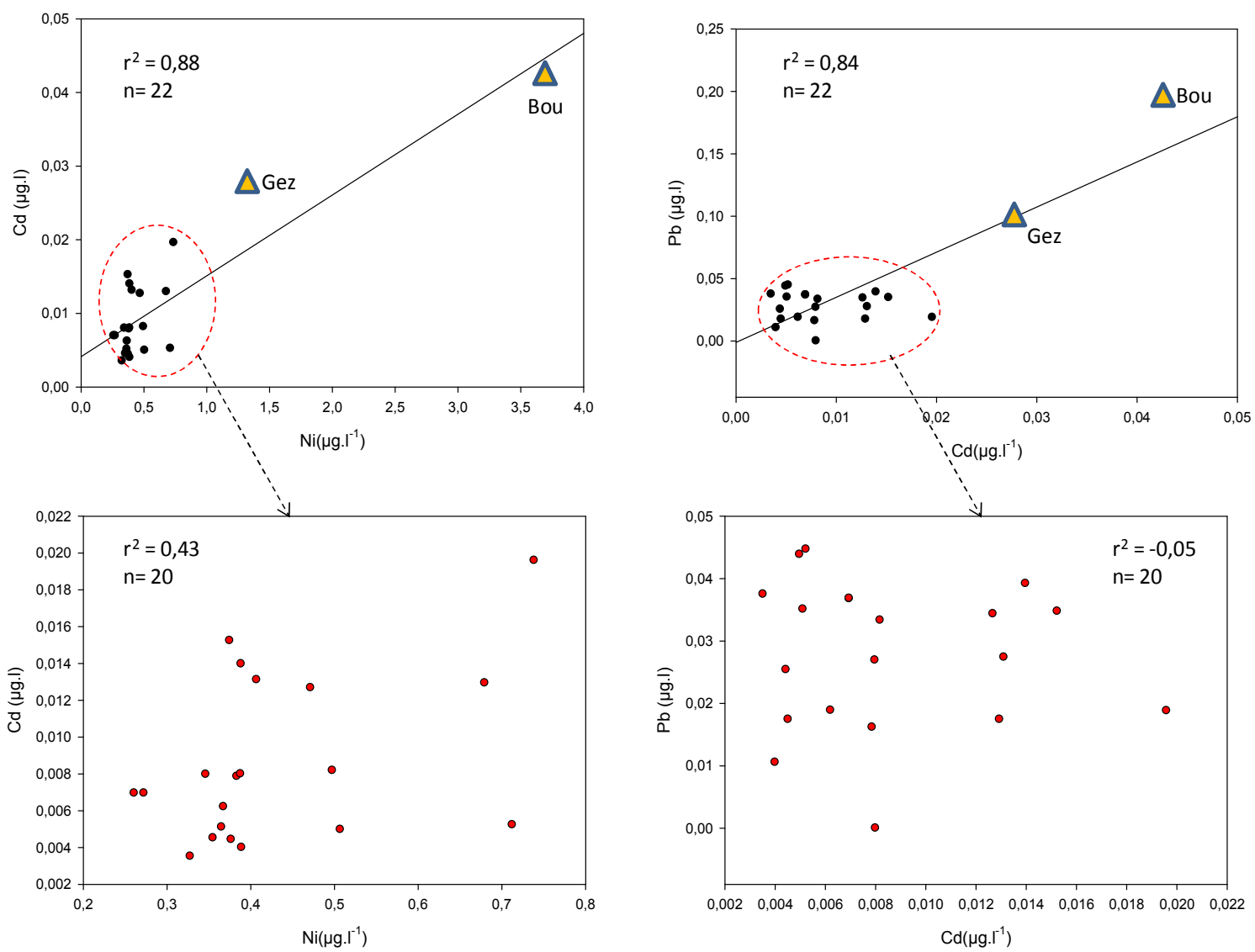

Figure 3 : Relation entre $\mathrm{Cd}, \mathrm{Ni}$ et $\mathrm{Pb}$ de la phase dissoute des affluents de la Garonne. 


\section{DISCUSSION}

Capacité de transport des éléments traces dans les affluents de la Garonne

Les résultats précédents ont montré que les concentrations en éléments traces des rivières de la région sont pour la plupart inférieures à celles des eaux naturelles du monde. Ces observations pourraient nous conduire à retenir que les eaux de la région sont faiblement ou pas du tout contaminées. Cependant, puisque les concentrations retrouvées dans la phase dissoute sont généralement négligeables par rapport à celles des sédiments de fond (Brunel, 2005), on peut supposer que les teneurs mesurées dans la colonne d'eau ne reflètent pas nécessairement le degré de pollution et la qualité environnementale d'un système aquatique. La preuve, dans les résultats précédents partant sur la variabilité spatiale des teneurs en éléments traces (Figure 2), on a observé une dispersion de $\mathrm{Cd}$ assez surprenante. En effet, parmi les échantillons analysés, les teneurs les plus faibles de $\mathrm{Cd}$ se trouvent au niveau des stations à fortes activités agricoles (Vem, Mar). En revanche, des stations comme « Bou » et « Gez » (avec une couverture forestière plus élevée) présentent les plus fortes teneurs en cadmium. De par son caractère anthropique, notamment son association dans les engrais phosphatés, le contraire de ces observations aurait pu être envisagé.

La comparaison (Tableau 2) des concentrations en éléments traces des affluents de la Garonne avec les différentes valeurs de l'Index de Transport Dissous (ITD), montre que les éléments les plus abondants dans la phase dissoute $(\mathrm{Zn}, \mathrm{As}, \mathrm{Cu})$ sont ceux qui possèdent des ITD élevés. Puisque l'ITD est l'expression de la capacité d'un élément à être transporté sous la forme dissoute, nous pouvons estimer que l'ordre d'abondance des éléments traces est logique. Le cas de $\mathrm{Zn}$ est à souligner, car malgré un ITD 2,5 fois inférieur à celui de As, il est cependant 4 fois plus concentré que l'arsenic. Ceci serait probablement lié à sa très grande abondance dans la roche mère locale, comparée à l'arsenic (Bur et al., 2009).
Les paramètres environnementaux influençant la mobilité des éléments traces

Les disparités observées dans la distribution des éléments traces pourraient être liées à l'action de la couverture végétale, ou plutôt à celle des paramètres physicochimiques de la phase dissoute.

L'action forestière est moins évidente car les stations SA1 et Bad, également sous forêt et situées dans la même zone géographique (Sud-Ouest) que « Gez », présentent pour certains éléments $(\mathrm{Ni}, \mathrm{Pb}$ et Cd) des concentrations 2 fois moins importantes que celles de « Gez ».

L'action des paramètres physicochimiques ( $\mathrm{pH}$, alcalinité, $\mathrm{HNO}_{3}{ }^{-}$et $\mathrm{SO}_{4}{ }^{2-}$ ) est probablement à l'origine des distributions spatiales des teneurs en éléments traces de la phase dissoute. En effet, les travaux de N'guessan et al. (2016) ont mis en évidence de fortes teneurs en $\mathrm{Cl}^{-}$et $\mathrm{COD}$ au niveau de la station («Bou »). Cette dernière présente également les plus fortes concentrations en éléments traces.

Pour vérifier l'influence exacte de ces paramètres physico-chimiques sur les polluants de l'ensemble des échantillons, l'importance de leurs associations a été évaluée.

Les résultats de ces relations sont résumés dans le Tableau 5. Globalement, les relations entre les éléments traces et les paramètres $\left(\mathrm{pH}\right.$, alcalinité, $\mathrm{HNO}_{3}{ }^{-}$et $\left.\mathrm{SO}_{4}{ }^{2-}\right)$ dont les teneurs sont souvent représentatives de l'occupation des sols et de la nature lithologique de la région, ne sont pas assez significatives $\left(r^{2}\right.$ compris entre $-0,45$ et 0,43$)$. En revanche, les relations avec $\mathrm{Cl}^{-}$et COD sont très significatives $\left(\mathrm{r}^{2}\right.$ compris entre 0,60 et 0,95), excepté Cr. Cependant, comme précédemment, lorsqu'on isole «Bou », toutes ces relations sont modifiées. $\mathrm{COD}$ et $\mathrm{Cl}^{-}$ deviennent moins corrélés aux éléments traces (excepté Co). A l'inverse, les relations avec les autres paramètres de l'eau deviennent significatives (Tableau 4), notamment pour $\mathrm{Cd}$ et $\mathrm{Pb}$. 
Tableau 4 : Coefficient de corrélation $\left(\mathrm{r}^{2} ; \mathrm{n}=23 ; \mathrm{p}<0,001\right)$ entre les éléments traces et les paramètres physico-chimiques.

\begin{tabular}{|c|c|c|c|c|c|c|c|c|}
\hline & $\mathrm{Cr}$ & Co & $\mathbf{N i}$ & $\mathbf{C u}$ & Zn & As & Cd & $\mathbf{P b}$ \\
\hline $\mathrm{Cl}$ & $0,25 / 0,18^{*}$ & $0,87 / 0,58 *$ & $\mathbf{0 , 8 6} / 0,04^{*}$ & $\mathbf{0 , 6 9} / 0,30$ & $0,31 /-0,27^{*}$ & $\mathbf{0 , 6 8} / 0,13^{*}$ & $\mathbf{0 , 6 9} /-0,01^{*}$ & $\mathbf{0 , 6 7 / - 0 , 3 7 ^ { * }}$ \\
\hline COD & $0,17 /-0,01 *$ & $\mathbf{0 , 9 5 / 0 , 8 4 *}$ & $\mathbf{0 , 8 6} / 0,18^{*}$ & $\mathbf{0 , 7 3} / 0,42 *$ & $0,48 / 0,17 *$ & $\mathbf{0 , 6 0} /-0,06^{*}$ & $\mathbf{0 , 6 0} /-0,23^{*}$ & $\mathbf{0 , 7 1} /-0,27^{*}$ \\
\hline $\mathrm{HCO}_{3}$ & $0,26 / 0,30^{*}$ & $0,02 / 0,28^{*}$ & $-0,30 /-0,41^{*}$ & $0,05 / 0,09 *$ & $-0,27 /-0,21^{*}$ & $0,08 / 0,30^{*}$ & $-0,46 /-\mathbf{0 , 5 2}$ * & $-0,45 /-\mathbf{0}, \mathbf{6 2}$ \\
\hline $\mathrm{SO}_{4}$ & $0,38 / 0,34^{*}$ & $0,34 / 0,08^{*}$ & $0,28 /-0,22 *$ & $0,43 / 0,28 *$ & $-0,08 /-0,30^{*}$ & $0,36 / 0,15^{*}$ & $0,18 /-0,17^{*}$ & $0,04 /-\mathbf{0 , 6 4}$ * \\
\hline $\mathrm{pH}$ & $0,06 / 0,14^{*}$ & $-0,05 / 0,35^{*}$ & $-0,33 /-0,33 *$ & $0,11 / 0,39 *$ & $-0,34 /-0,26^{*}$ & $0,11 / 0,44 *$ & $-0,41 / \mathbf{- 0 , 5 8 *}$ & $-0,44 /-\mathbf{0}, \mathbf{5 0}$ \\
\hline
\end{tabular}

Ces résultats mettent en évidence le contrôle des éléments traces de la phase dissoute par les chlorures et la matière organique lorsque les concentrations de ces derniers sont très importantes (environ 5meq. $\mathrm{l}^{-1}$ et $8 \mu \mathrm{g} . \mathrm{l}^{-1}$, respectivement). En revanche, pour de faibles teneurs de ces ligands, les concentrations des éléments traces (notamment $\mathrm{Cd}$ et $\mathrm{Pb}$ ) sont plus ou moins influencées par le $\mathrm{pH}$ et l'alcalinité. Les valeurs de ces paramètres, liées à la nature lithologique de la région, évoluent inversement aux teneurs en éléments traces de la phase dissoute. Ceci signifie que lorsque les eaux de la région deviennent de plus en plus basique, moins il y a de polluants métalliques (notamment $\mathrm{Cd}$ et $\mathrm{Pb}$ ) dans la phase dissoute. Toutefois, lorsque les concentrations de certains ligands (notamment la matière organique et les chlorures) augmentent dans la phase dissoute, grâce aux apports anthropiques, les éléments traces sont complexés par ces derniers, et l'influence des autres paramètres devient moins évidente.

\section{Conclusion}

Les concentrations des éléments traces métalliques dissouts des affluents du fleuve Garonne évoluent en moyenne de $0,01 \mu \mathrm{g} . l^{-1}$ (Cd) à 4,46 $\mu \mathrm{g} \cdot \mathrm{l}^{-1}(\mathrm{Zn})$. L'ordre d'abondance de ces polluants s'établit comme suit: $\mathrm{Zn}>\mathrm{As}$ $>\mathrm{Cu}>\mathrm{Ni}>\mathrm{Cr}>\mathrm{Co}>\mathrm{Pb}>\mathrm{Cd}$. Le $\mathrm{Pb}$ et le $\mathrm{Cd}$, avec des teneurs respectives de $0,04 \mu \mathrm{g} \cdot 1^{-1}$ et $0,01 \mu \mathrm{g} . \mathrm{l}^{-1}$, sont en moyenne plus de 10 fois moins concentrés que les autres éléments traces. Dans l'ensemble, les plus fortes concentrations en éléments traces des phases dissoutes de la région se rencontrent au niveau de la station «Bou » située sur la rivière Rieu Tord, à l'exception de $\mathrm{Zn}$ et de $\mathrm{Cr}$ qui présentent, respectivement, leur maxima à « $\mathrm{TO} 2$ » (rivière Touch) et à « Vem » (rivière vermeil).

La distribution des éléments traces dissous, dans la région Gascogne, est contrôlée par les chlorures et le carbone organique dissous, notamment dans les zones où les apports anthropiques sont importants, augmentant ainsi la mobilité et la disponibilité de ces polluants. Par contre, dans les zones moins anthropisées, la distribution des éléments traces (notamment $\mathrm{Pb}$ et $\mathrm{Cd}$ ) évolue inversement au $\mathrm{pH}$ et à l'alcalinité de l'eau, créant ainsi une "filtration naturelle" de la colonne d'eau. Aucune action notable des activités agricoles n'a été révélée dans les teneurs de ces éléments dans la phase dissoute.

\section{CONFLITS D'INTERETS}

Les auteurs déclarent l'absence de tout conflit d'intérêts relatif à cet article.

\section{CONTRIBUTIONS DES AUTEURS}

YMN a réalisé le prélèvement et l'analyse des échantillons, a conçu et rédigé le manuscrit; VAW et KGK ont participé à la rédaction et à la correction du manuscrit; $\mathrm{AP}$ et JLP ont contribué à l'analyse des échantillons et la rédaction du manuscrit. 


\section{REMERCIEMENTS}

Nos remerciements vont à l'endroit des techniciens du Laboratoires d'Ecologie Fonctionnelle (ECOLAB) et du Laboratoire des Mécanismes et des Transferts en Géologie (LMTG) de Toulouse, notamment G. Durbe et A. Alric pour leurs contributions dans l'échantillonnage, ainsi que dans la préparation et l'analyse des échantillons.

\section{REFERENCES}

Aduayi-Akue AA, Gnandi K. 2014. Evaluation de la pollution par les métaux lourds des sols et de la variété locale du maïs Zea mays dans la zone de traitement des phosphates de Kpémé (Sud du Togo). International Journal of Biological and Chemical Sciences, 8(5): 2347-2355.

DOI: http://dx.doi.org/10.4314/ ijbcs.v8i5.37

Baque B. 2006. Perturbations anthropiques du réseau hydrographique du bassin de la Garonne, cas des métaux et des nitrates. Thèse de doctorat, Toulouse III, p. 476.

Bliefert C, Perraud R. 2001. Chimie de l'Environnement : Air, Eau, Sols, Déchets. De Boeck: Bruxelles.

Brunel C. 2005. Dynamique des éléments traces métalliques $(\mathrm{Pb}, \mathrm{Zn}, \mathrm{Cd})$ sur un petit bassin versant amont contaminé par des déchets miniers - cas du bassin versant amont du Lez (Ariège, Pyrénées). Thèse de doctorat, Université de Toulouse 3, p. 267.

Bur T, Probst JL, N'guessan M, Probst A. 2009. Distribution and origin of lead in stream sediments from small agricultural catchments draining Miocene molassic deposits (SW France). Applied Geochemistry, 24(7): 1324-1338. DOI: https://doi.org/10.1016/j.apgeochem.200 9.04.004

Devallois V. 2009. Transferts et mobilité des éléments traces métalliques dans la colonne sédimentaire des hydrosystèmes continentaux. Thèse de doctorat, Université de Provence, Section sciences, p. 277.
Kabata-Pendias A, Mukherjee AB. 2007. Trace elements from soil to human. Springer Science and Business Media.

Martin JM, Meybeck M. 1979. Elemental mass-balance of material carried by major world rivers. Marine Chemistry, 7(3): 173-206. DOI : https://doi.org/10.1016/03044203(79)90039-2

Mélila M, Poutouli W, Amouzou KS, Tchangbédji G, Tchaou M, Doh A, Goto C. 2012. Induction du stress oxydatif chez l'homme suite à la bioconcentration des éléments métalliques (cadmium et plomb) par voie trophique à Kpémé (Sud $\mathrm{du}$ Togo). International Journal of Biological and Chemical Sciences, 6(3): 1263-1270.

DOI: http://dx.doi.org/10.4314/ ijbcs.v6i3.29

Meybeck M, Helmer R. 1989. The quality of rivers: from pristine stage to global pollution. Global and Planetary Change, 1(4): 283-309. DOI: https://doi.org/10.1016/ 09218181(89)90007-6

N'guessan YM, Probst JL, Bur T, Probst A. 2009. Trace elements in stream bed sediments from agricultural catchments (Gascogne region, S-W France): Where do they come from? Science of the Total Environment, 407: 2939-2952. DOI: https://doi.org/10.1016/j.scitotenv.2008. 12.047

N'guessan YM, Wango TE, Adopo KL, Probst JL, Probst A. 2016. Caractéristiques géochimiques des eaux de surface dans un environnement agricole : cas des bassins versants de la Gascogne (Région Midi Pyrénées, S-W de la France). International Journal of Innovation and Applied Studies, 17(2): 394-406. DOI: http://www.ijias.issrjournals.org/abstract.php?article=IJIAS16-139-04

Oeurng C, Sauvage S, Sánchez-Pérez JM. 2010. Dynamics of suspended sediment transport and yield in a large agricultural catchment, southwest France. Earth 
Surface Processes and Landforms, 35(11), 1289-1301. DOI: https://doi.org/ 10.1016/j.ecolind.2011.10.002.

Pekey H. 2006. The distribution and sources of heavy metals in Izmit Bay surface sediments affected by a polluted stream. Marine Pollution Bulletin, 52: 1197 1208. DOI: https://doi.org/10.1016/ j.marpolbul.2006.02.012

Perrin AS, Probst A, Probst JL. 2008. Impact of nitrogenous fertilizers on carbonate dissolution in small agricultural catchments: Implications for weathering $\mathrm{CO} 2$ uptake at regional and global scales. Geochimica et Cosmochimica Acta, 72(13): 3105-3123. DOI: https://doi.org/10.1016/j.gca.2008.04.01 1

Quevauvillier P. 2001. Métrologie en Chimie de l'Environnement. Editions Tec \& Doc.

Roussiez V, Ludwig W, Probst JL, Monaco A. 2005. Background levels of heavy metals in surfacial sediments of the Gulf of Lions (NW Mediterranean): An approach based on 133Cs normalization and lead isotope measurements. Environmental Pollution, 138: 167-177.
DOI:

https://doi.org/10.1016/j.envpol.2005.02. 004

Senou I, Gnankambary Z, Some AN, Sedogo MP. 2012. Phytoextraction du cadmium, du cuivre, du plomb et du zinc par Vetiveria nigritana en sols ferrugineux tropicaux et en sols vertiques au Burkina Faso (Afrique de l'ouest). International Journal of Biological and Chemical Sciences, 6(4): 1437-1452. DOI: http://dx.doi.org/10.4314/ijbcs.v6i4.4

Soro G, Metongo SE, Soro N, Ahoussi KE, Kouame KF, Zade SGP, Soro T. 2009. Métaux lourds $(\mathrm{Cu}, \mathrm{Cr}, \mathrm{Mn}$ et $\mathrm{Zn})$ dans les sédiments de surface d'une lagune tropicale africaine : cas de la lagune Ebrie (Côte d'Ivoire). International Journal of Biological and Chemical Sciences, 3(6): 1408-1427. DOI: http://dx.doi.org/10.4314/ijbcs.v3i6.5316 1

Taghavi L. 2010. Dynamique de transfert des pesticides en périodes de crue sur les bassins versants agricoles gascons. Thèse de doctorat, Université de Toulouse, p. 278. 